\title{
A CMOS Fully-Differential BandPass $\Sigma \Delta$ Modulator Using Switched-Current Circuits
}

\author{
J.M. de la Rosa, F. Medeiro, B. Pérez-Verdú and A. Rodríguez-Vázquez. \\ Dept. of Analog Design, Centro Nacional de Microelectrónica (CNM), Ed. CICA, Av. Reina Mercedes s/n, 41012 \\ Sevilla, SPAIN. Phone: (34)-5-4239923, Fax: (34)-5-4624506, E-mail: angel@.cnm.us.es
}

\begin{abstract}
This paper presents a fourth-order bandpass sigma-delta modulator that has been designed using fully-differential switched-current circuits in a $0.8 \mu \mathrm{m}$ CMOS technology. The modulator prototype has been obtained by applying a lowpass to bandpass transformation $\left(\mathrm{z}^{-1}->-\mathrm{z}^{-2}\right)$ to a second-order lowpass $\Sigma \Delta$ modulator. Specifications are $S N R \geq 60 \mathrm{~dB} @ 2.5 \mathrm{Mhz} \pm 15 \mathrm{Khz}$, for a clock frequency of $10 \mathrm{Mhz}$. Preliminary results from the fabricated prototype obtains the correct noise shaping up to 2.5Mhz clock frequency.
\end{abstract}

\section{INTRODUCTION}

Bandpass oversampled $\Sigma \Delta$ converters are very convenient for the front-end of modern narrow-band communication systems [1-3]. Similar to lowpass, bandpass $\Sigma \Delta$ converters use oversampling and feedback of the quantization noise to exchange operation speed for resolution and thus increase robustness against unavoidable hardware parasitics and tolerances.

Realizations of bandpass $\Sigma \Delta$ modulators using continuous-time circuits with off-chip LC resonators [4] and monolithic discrete-time switched-capacitor (SC) circuits [5-7] have been reported. These latter prototypes featured center frequencies in the range of $455 \mathrm{kHz}$ to $10.7 \mathrm{MHz}$, bandwidths in the range of $10 \mathrm{Khz}$ to $200 \mathrm{Khz}$, and resolutions in the range of 9 to $14 \mathrm{bit}$, in BiCMOS [7] or double-poly CMOS technology [5-6]. However, since standard CMOS have only one poly layer it may be worth exploring the use of switched-currents (SI), which require only MOS transistors as design primitives [8]. Besides, these techniques show promise for higher operation speed than switched-capacitors [9], although this is a rather controversial issue. Some preliminary results in this direction have been presented recently in $[10]$.

This paper presents the architecture and implementation of an SI 4th-order bandpass $\Sigma \Delta$ converter in a $0.8 \mu \mathrm{m}$ CMOS digital technology. The prototype occupies $1.2 \times 1.4 \mathrm{~mm}^{2}$ silicon area and the simulation results foresee 11.6 bit resolution for a signal of $2.5 \mathrm{Mhz} \pm$ $15 \mathrm{Khz}$ with $10 \mathrm{Mhz}$ sampling frequency.

0-7803-2972-4/96\$5.00@1996 IEEE

\section{Architecture of the Modulator}

Fig. 1 shows the architecture chosen for the modulator, determined from a second-order lowpass prototype using the transformation $z^{-1}>-z^{-2}[5]$. This transformation maintains the stability properties of the lowpass prototype, whose practical use has been demonstrated in both SC and SI implementations [11]. Assuming that the 1 bit quantizer in Fig. 1 can be modelled as an additional noise source with $k$ effective gain, the modulator output is given as,

$$
Y(z)=z^{-2} X(z)+\left(1+z^{-2}\right)^{2} E(z)
$$

where $X(z)$ is the modulator input signal, $Y(z)$ is the modulator output, and $E(z)$ is the additional quantization noise source in the linear model. The transfer function of the input is a double-delay and the transfer function of the quantization noise has two transmission zeros in $f_{s} 4$. This is the equivalent of the two zeros in $\mathrm{DC}$ of the lowpass prototype.

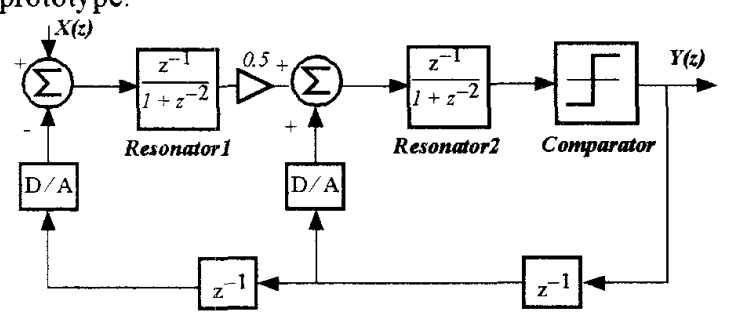

Fig. 1: Block Diagram of the Modulator

Fig. 2 presents the simulation results of the proposed architecture obtained using $A S I D E S$ [12], a simulator of the behavior level of $\Sigma \Delta$ modulators. Fig. 2a shows an FFT of the modulator output for a sinusoidal input of $-6 \mathrm{~dB}$ range centered on $2.5 \mathrm{Mhz}$ to a clock frequency of $10 \mathrm{Mhz}$. The coefficient 0.5 offers a similar dynamic range in the output of both resonators as shown in the histogram of Fig. $2 b$; thus their design can be identical.

\section{Design of the modulator blocks}

\section{A.Resonator SI}

The resonator block has been implemented using a 
cascade of two backward-Euler integrator blocks with biquad feedback [7] as shown in Fig 3. Making $A=B= \pm 1$ and $R=2$, obtains the desired transfer function. This structure has been chosen from others by stability reasons, since its poles remain in the circle unit upon changes in the $R$ coefficient.
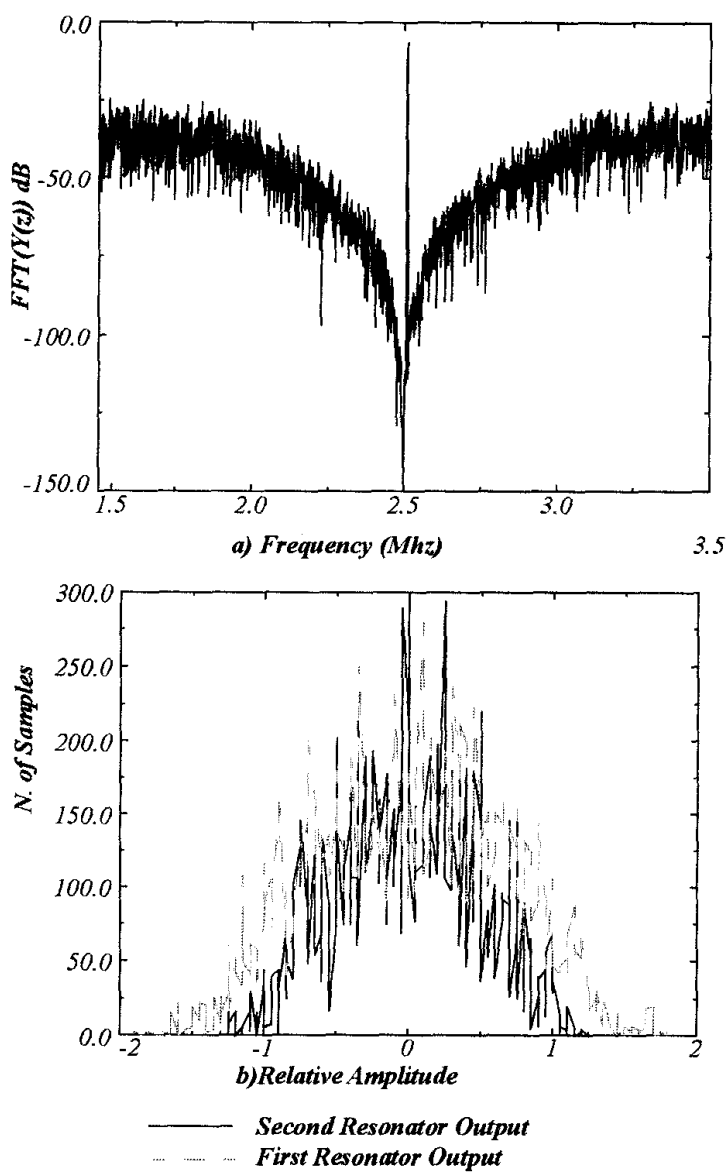

Fig. 2: Behavior Simulations of the Modulator

The implementation of the integrator with SI techniques is shown in Fig. 4. For maximum reduction of errors, we used a fully-differential regulated folded cascode structure [13]. The local feedback of this cell increases the input conductance and the differential structure allows first-order cancellation of the clock feedthrough. The common mode feedback $C M F B$ stage is shown as the thickest line. Delay versions have been used for the clock phases that control the steering switches. At the same time, minimal dummy transistors were used in the memory switches.

The gain $A$ is performed by scaling the memory transistors at the integrator output stage. Consequently, the coefficient $R$ is realized by adding an additional output stage to the second integrator with -2 gain as show schematically in Fig. 5.
The gain $A$ is performed by scaling the memory

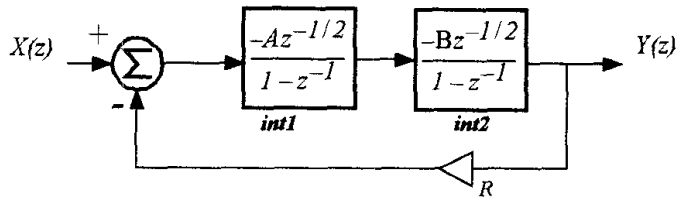

Fig. 3: Block Diagram of Resonator

transistors at the integrator output stage. Consequently, the coefficient $R$ is realized by adding an additional output stage to the second integrator with -2 gain as show schematically in Fig. 5.

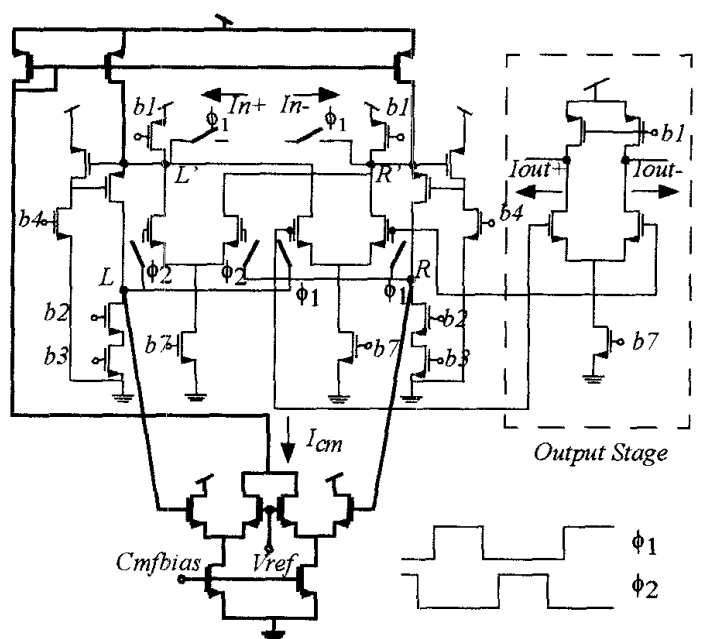

Fig. 4: Regulated Folded-Cascode Integrator with CMFB

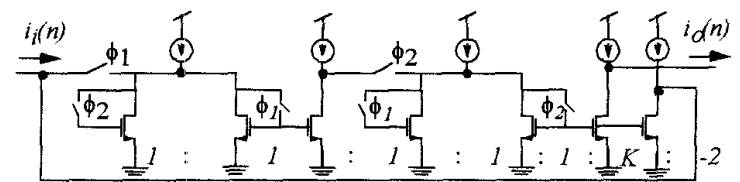

Fig. 5: Simplified Schematic of the SI Resonator

\section{B.1-bit D/A Converter}

Fig. 6 shows a schematic of the 1-bit D/A converter used, consisting of a current source controlled by the comparator output [11]. The type of current source used has been chosen so that it resembles the structure of the cell memory. The two output currents change the flow direction depending on the comparator output. A small offset is no serious problem for the modulator. $P$ mos switches are used clue to the input voltage range required in the integrators.

\section{C.1-bit Quantizer}

Fig. 7 shows the quantizer block diagram, made up of a regenerative latch [15] and an RS flip-flop that maintains 


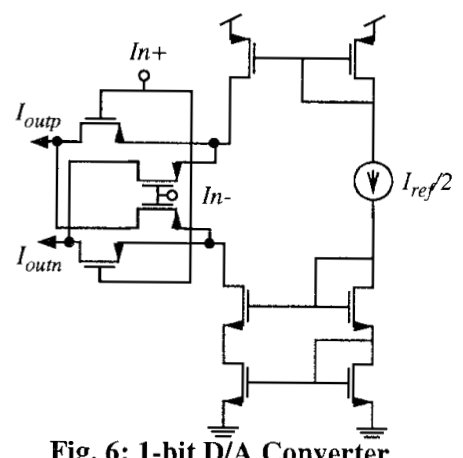

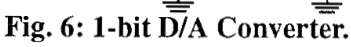

the output value in the phase $\phi_{1}$, which is the phase where the phase where the resonators are feedback.

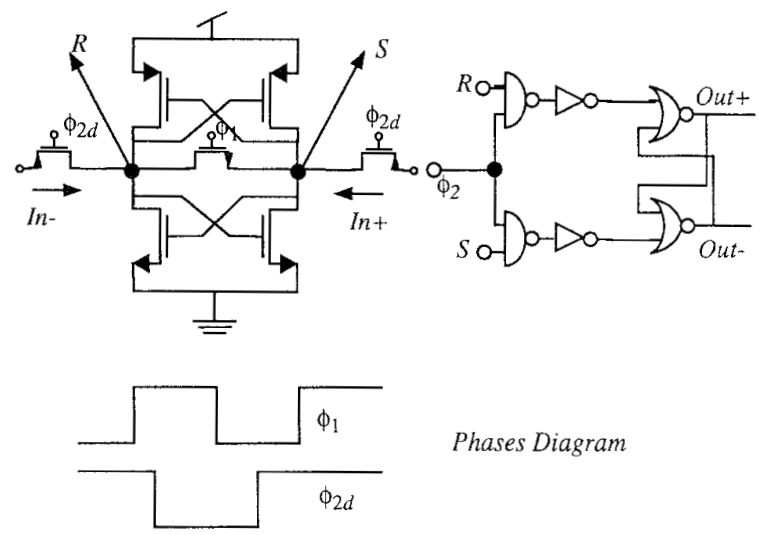

Fig. 7: Block diagram of the Current Quantizer

The regenerative latch operates as follows: the input currents are sampled in the complementary $\phi_{2 \mathrm{~d}}$ phase since the switches are pmos, developing a small difference voltage through the nmos switch. When the nmos switch opens, the current which flows through this switch cuts off abruptly, and the latch impedance changes from positive to negative (positive feedback). Consequently, depending on the sign of the small difference of stored voltage, the latch output nodes ( $R$ and $S$ ) will go to the positive or negative rail. A quick comparison is thus obtained with low input current levels.

\section{Simulation Results}

This section presents some results of the electrical simulation obtained throughout the design. Fig. 8 shows several simulations made of the modulator blocks using HSPICE. Fig. 8a shows an analysis in AC of the cell memory, it presents $50 \mathrm{Mhz}$ bandwidth. Transient simulation of this cell in the storage phase is shown in Fig. $8 \mathrm{~b}, v($ out $)$ is the gate voltage of the memory transistor, Fig. $8 \mathrm{c}$ shows the time response of the integrator to a sinusoidal input of $5 \mu \mathrm{A}$ range and $1 \mathrm{Mhz}$ when sampled to $10 \mathrm{Mhz}$. Finally, Fig. 8d shows a simulation of the time response of the Resonator block when it is excited by an impulse signal of $10 \mu \mathrm{A}$ range. The Resonator output should repeat the values in the ranges $10 \mu \mathrm{A}, 0,-10 \mu \mathrm{A}, 0$ periodically. Since
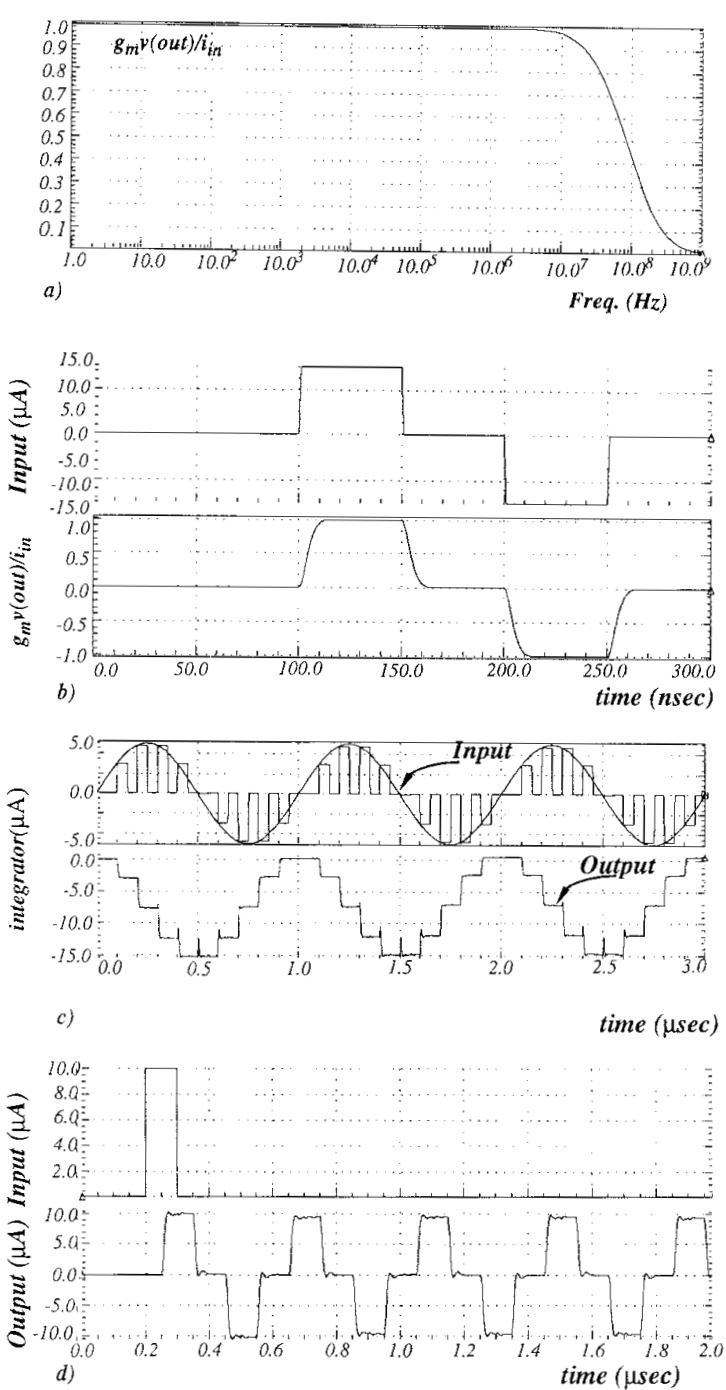

Fig. 8: HSPICE Simulations of the Memory Cell and Resonator.

there are loss coefficients, the output range value attenuates.

Fig. 9 shows simulation of the $S N R$ regarding the input range, taking into account the distinct non-linearities appearing in SI circuits. These have been measured using HSPICE and introduced in the ASIDES models for the modulator blocks. The values of these errors are: finite conductance ratio error $e_{g}=0.04 \%$, settling error $e_{s}=0.27 \%$, and load injection error $e_{q}=0.23 \%$. A variation of $1.0 \%$ in the value of $R$ has also been considered.

\section{Conclusions}

An SI bandpass $\Sigma \Delta$ modulator has been designed up to a layout level in a CMOS $0.8 \mu \mathrm{m}$ technology of AMS. The arquitecture is based in a second order lowpass prototype using the transformation $z^{-1}->-z^{-2}$. The SI cell memory used are fully differential regulated cascode to minimize the 
errors. The predicted resolution is of 11.6 bits for a sampling frequency of $10 \mathrm{Mhz}$ applied to a signal centered on $2.5 \mathrm{Mhz}$ with a bandwidth of $30 \mathrm{Khz}$. Fig. 11 shows the entire Layout of the circuit, including the pads with an area of $1.2 \times 1.4 \mathrm{~mm}^{2}$. In addition to the modulator, a clock phase generator and a single-ended V-I to differential converter have been included to ease testing, as shown in Fig. 10. A preliminary experimental result from the fabricated prototype is shown in Fig. 12. This spectrum corresponds to the output of the bandpass modulator with $2.5 \mathrm{Mhz}$ of clock frequency and a $-8.2 \mathrm{~dB}$ sinusoidal input.The functionality of the SI bandpass is demonstrated, and better resolution and frequency are expected.

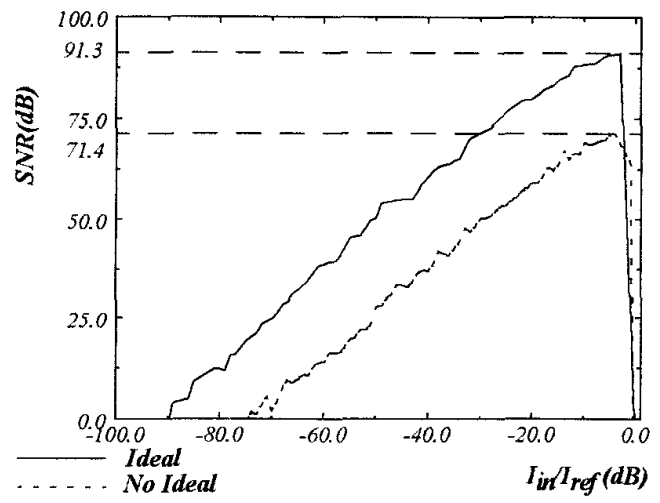

Fig. 9: SNR vs. relative range

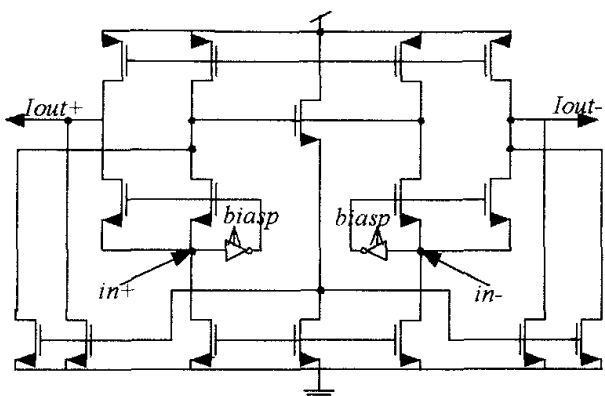

Fig. 10: Input Buffer

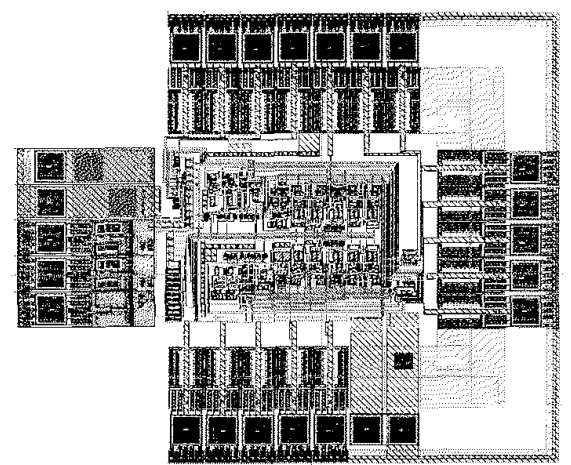

Fig. 11: Complete Layout of the Modulator.

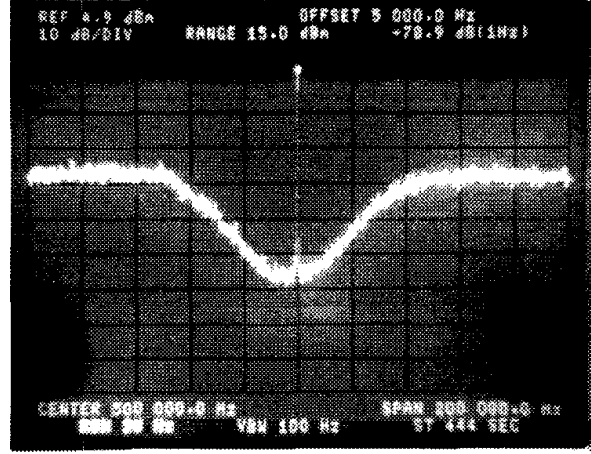

Fig. 12: Preliminary Experimental Result.

\section{References}

[1]A.M. Thurston, T.H. Pearce, M.D. Higman, M.J. Hawksford, "Bandpass Sigma Delta A-D Conversion", Workshop on Advances in Analog Circuit Design, pp. 267-297, April 1992.

[2]R. Schreier and M. Snelgrove, "Bandpass Sigma-Delta Modulation", Electronics Letters, Vol. 25,pp. 1560-1561, November 1989.

[3]S. Jantzi, Schreier, and M. Snelgrove, "Bandpass sigma-delta analog-to-digital Conversion", IEEE Trans. Circuits and Systems, Vol. 31, pp. 1406-1409, November 1991.

[4]G. Troster, P. Sieber, K. Schoppe, A. Wedel, E. Zocher and J. Amdt "An interpolative bandpass converter on a 1.2 um BiCMOS analog/digital array", IEEE J. Solid State Circuits, Vol. 28, pp. 471-477, April 1993.

[5]L. Longo and H. Bor-Rong, "A $15 b$ 30Khz bandpass sigma-delta Modulator", Proceedings of the 1993 IEEE Int. Solid-State Circuits Conf., pp. 226-227, March 1993.

[6]S.A. Jantzi, M. Snelgrove and P.F. Ferguson, "A fourth-order Bandpass sigma-delta Modulator", IEEE J. Solid-State Circuits, Vol. 28, pp. 282-291, March 1993.

[7]F.W. Singor and W. M. Snelgrove, "Switched-Capacitor Bandpass Delta-Sigma A/D Modulation at $10.7 \mathrm{Mhz}$ ", IEEE J. Solid-State Circuits, Vol.30,pp. 184-192, March 1995

[8]C. Toumazou, J.B. Hughes and N.C. Battersby, Analogue IC Design the current-mode approach, London: Peter Peregrinus, 1990

[9]J.B. Hughes and K.W. Moulding "Switched-Current Signal Processing for Video Frequencies and Beyond", IEEE J. Solid-State Circuits, Vol.28,pp. 314-322, March 1993.

[10]S.V. Pattamatta, P. Manapragada, V. Dalal and R. Schreier, "A switched current Bandpass Delta sigma modulator", Proc. 199. IEEE Int. Symp. Circuits and Systems, pp. 477-480, May 1994.

[11]N. Tan, "Oversampling AD Converters and Current-Mode Techniques", PhD Dissertation, Linkoping University, 1994.

[12]F.Medeiro, B. Pérez Verdú, A. Rodriguez Vázquez,and J.L. Huertas, "A Vertically-Integrated Tool for Automated Design of $\Sigma \triangle$ Modulators". IEEE Journal of Solid-State Circuits, Vol. 30, pp. 762-772, July 1995.

[13]M. Goldenberg, R. Croman, and T. S. Fiez, "Accurate SI Filters Using RGC Integrators", IEEE J. Solid-State Circuits, Vol. 29, pp. 1388-1395, November 1994.

[14]J.F. Duque-Carrillo, "Control of the Common-Mode Component in CMOS Continuous-Time Fully Differential Signal Processing", Analog Integrated Circuits and Signal Processing, Vol. 4, pp. $131-140,1993$

[15]M. Bracey and W. Redman-White, "Design Considerations for Current Domain Regenerative Comparators", Advanced A-D and D-A Conversion Techniques and their Applications, pp.65-70, July 1994 